\title{
EL CONSUMO DE ALIMENTOS TRANSGÉNICOS (ATS) EN EL SUR DE MANABÍ, ECUADOR
}

\author{
AUTORES: Julio Gabriel Ortega ${ }^{1}$ \\ Cinthia Carchi Carch² \\ Karla Morán Nieto ${ }^{3}$ \\ Lizeth Salazar Salazar ${ }^{4}$ \\ Paola Castro Quijije ${ }^{5}$
}

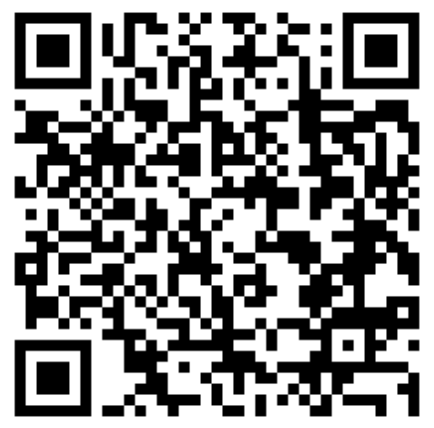

\section{DIRECCIÓN PARA CORRESPONDENCIA:julio.gabriel@unesum.edu.ec}

Fecha de recepción: 12/01/2020

Fecha de aceptación: 05/02/2020

\section{RESUMEN}

Con los objetivos de: i) determinar la presencia de alimentos transgénicos (ATs) en cadenas de supermercados y negocios, ii) conocer la precepción de la sociedad civil acerca de los ATs y iii) determinar la percepción de la comunidad universitaria acerca de los ATs; fueron realizados visitas a los centros comerciales como el Comisariato, AKI, TIA y tiendas de barrio, en Jipijapa, Porto Viejo y Manta. También se realizaron encuestas de 13 preguntas a 250 consumidores de la sociedad civil y 425 encuestas a la comunidad universitaria de la UNESUM. Los resultados mostraron que $40 \%$ de los jóvenes conocen los ATs y más del 50\% saben identificarlos. Pudimos inferir que el nivel de compresión y conocimiento sobre los alimentos transgénicos en el Ecuador ha cambiado en los últimos años. Se constató que ambos grupos encuestados consideran que los ATs son importantes para su venta por los intereses económicos, por presión internacional y por la petición de los consumidores. Por otra parte, la percepción de ambos grupos fue que los ATs son

\footnotetext{
${ }^{1}$ Profesor investigador, Carrera Agropecuaria, Facultad de Ciencias Naturales y de la Agricultura, Universidad Estatal del Sur de Manabí, Km 11⁄2 vía Jipijapa-Noboa - Campus los Ángeles.

${ }^{2}$ Estudiantes de las asignaturas de Genética y Bioestadística de Quinto nivel, Carrera Agropecuaria, Facultad de Ciencias Naturales y de la Agricultura, Universidad Estatal del Sur de Manabí, Km 1²̌2 vía Jipijapa-Noboa - Campus los Ángeles.

${ }^{3}$ Estudiantes de las asignaturas de Biología Molecular de Cuarto nivel, Carrera Agropecuaria, Facultad de Ciencias Naturales y de la Agricultura, Universidad Estatal del Sur de Manabí, Km 11/2 vía Jipijapa-Noboa - Campus los Ángeles.

${ }^{4}$ Estudiantes de las asignaturas de Biología Molecular de Cuarto nivel, Carrera Agropecuaria, Facultad de Ciencias Naturales y de la Agricultura, Universidad Estatal del Sur de Manabí, Km 1²/2 vía Jipijapa-Noboa - Campus los Ángeles.

${ }^{5}$ Estudiantes de las asignaturas de Biología Molecular de Cuarto nivel, Carrera Agropecuaria, Facultad de Ciencias Naturales y de la Agricultura, Universidad Estatal del Sur de Manabí, Km 1¹/2 vía Jipijapa-Noboa - Campus los Ángeles.
} 
Julio Gabriel Ortega, Cinthia Carchi Carch, Karla Morán Nieto...

beneficiosos para el consumo, pero al preguntar sobre los beneficios o perjuicios de los ATs indicaron que estos son perjudiciales para la salud y el medioambiente. Además, la sociedad civil considera de mayor importancia a los ATs porque son más baratos, y la comunidad universitaria opinó que lo más importante es la mayor conservación de los ATs. Finalmente ambos grupos mencionaron que si comprarían los ATs y que tienen el derecho de decidir para consumirlos; y, ambos grupos enfatizaron que las empresas están en la obligación de que sus productos y/o ATs deben presentar etiquetas visibles. Fue notorio la alta significancia al $\mathrm{P}<0.01$ de probabilidad de los criterios en las 13 preguntas realizadas en cada pregunta y en cada grupo, denotando esto que al menos un criterio fue diferente.

\section{PALABRAS CLAVE: Encuestas, sociedad, comunidad, consumidor, biotecnología. \\ THE CONSUMPTION OF TRANSGENIC FOODS (TFS) IN SOUTH OF MANABÍ, ECUADOR}

\section{ABSTRACT}

With the objectives of: i) determining the presence of Trangenic Foods (FTs) in supermarket and business chains, ii) knowing the civil society's preceptions about FTs and iii) determine the perception of the university community about ATs; visits were made to shopping centers such as the Comisariato, AKI, TIA and neighborhood stores, in Jipijapa, Porto Viejo and Manta. So, surveys were conducted with 13 questions to 250 consumers of civil society and 425 surveys to the university community of UNESUM. The results showed that $40 \%$ of young people know about TFs and more than $50 \%$ know how to identify them. We could infer that the level of compression and knowledge about TFs in Ecuador has changed in recent years. We were able to determine that both groups surveyed consider that Tfss are important for sale due to economic interests, international pressure and the request of consumers. On the other hand, the perception of both was that TFs are beneficial for consumption, but when asked about the benefits or harms of TFs, they indicated that they are harmful to health and the environment. In addition, civil society considers TFs to be of greater importance because they are cheaper, and the university community said that the most important thing is the greater conservation of TFs. Finally, both groups mentioned that they would buy the TFs and that they have the right to decide to consume them; and, both groups emphasized that companies are under the obligation that their products and/or TF must present visible labels. The high significance at $\mathrm{P}<0.01$ of the probability of the criteria in the 13 questions asked in each question and in each group was noted, indicating that at least one criterion was different.

KEYWORDS: Surveys, society, community, consumer, biotechnology

\section{INTRODUCCIÓN}

Antes de entrar en el tema de investigación en cuestión, convendría entender que es un Organismo Transgénico (OT) y qué es un Organismo Genéticamente Modificado (OGM). Ambos términos son utilizados erróneamente como sinónimos, tal como lo especifican diversos investigadores (Gabriel 2014, Gabriel et al. 2018, Petit Glossaire 2019, Europan Comission, 2019). Un OT es un organismo cuyo material genético fue alterado artificialmente en laboratorio, usando la tecnología de ADN recombinante (ingeniería genética); en cambio un OGM es un organismo que se obtiene naturalmente o mediante uso de métodos convencionales de mejora genética (Gabriel et al., 2018). 
En el presente artículo, con propósitos prácticos no haremos controversia sobre ambas terminologías y nos referiremos a los OTs y OGMs como sinónimos, pero considerando que se hará referencia básicamente a los alimentos transgénicos (ATs) que fueron producto de OTs.

En la actualidad, existen fuertes controversias entre quienes promueven y quienes son detractores de la producción de OGMs, en relación a su conveniencia, seguridad e impacto sobre el medioambiente y el hombre. Se debe reconocer que la práctica de modificar genéticamente las especies para uso humano acompaña a la humanidad desde sus orígenes (Scott, 2013), aunque muy recientemente se realiza en laboratorios y no en el campo o zonas de cultivo directamente. Sin embargo, la inocuidad de los transgénicos en el ambiente es objeto de controversia entre los sectores a favor de esta clase de biotecnología y los sectores ambientalistas en contra de la misma. Ambos sectores argumentan estudios científicos para sustentar sus posturas, y se acusan mutuamente de ocultar - o ignorar - hechos frente al público (Chilebio, 2019).

Según Oleas et al. (2016). los avances biotecnológicos alcanzados en las últimas décadas aplicados en la industria de alimentos permitieron desde hace más de 15 años la creación de alimentos genéticamente modificados, cuya presencia generó controversia; creando desconfianza, debate social y actitudes intensamente negativas entre los consumidores, especialmente de países desarrollados de Europa y Japón (Gaskell et al. 2006). El principal motivo que generó aversión a los alimentos transgénicos (ATs) en los consumidores, es el presunto riesgo para la salud, situación que predispone a evitar su consumo y a pagar un mayor precio por otros alimentos no transgénicos (Zilberman et al. 2013).

A pesar de la resistencia a los AT, según el último informe del ISAAA (Servicio para la Adquisición de Aplicaciones Agro-biotecnológicas, 2019), 18 millones de agricultores de 26 países sembraron cultivos genéticamente modificados (GM) en 185,1 millones de hectáreas en 2016. Esta superficie representa un aumento neto de 3\% respecto de las 179,7 millones de hectáreas sembradas con OGM a nivel global en 2015 (Argenbio, 2019 ISAAA, 2019). Es decir, que la producción de ATs se incrementó de gran manera, lo que llevó a crear un mayor ambiente de controversia entre consumidores, que por lo general se muestran mal informados sobre el tema (Oleas et al. 2016). Sin embargo, gran parte de los investigadores actualmente manifiestan que se dispone de instrumentos para garantizar el proceso de modificación genética de los alimentos y que los riesgos supuestos son mínimos. Aun así, la preocupación entre los consumidores se mantiene debido a la falta de estudios acerca de los efectos de los ATs en el medioambiente (Jurkiewicz et al., 2014). Se realizó estudios de opinión mediante encuestas en varios países como Argentina, Chile (Traynor et al. 2007), Estados Unidos, y Canadá, así como en algunos estados miembros de la Comunidad Europea, entre ellos España y Francia, para conocer la opinión de los consumidores sobre los ATs (European Commission, 2010; Evans et al. 2013). Los resultados obtenidos difieren de un país a otro. Mientras que países de la Unión Europea son muy críticos con los cultivos transgénicos, otros países son indiferentes o se muestran a favor de los ATs y productos relacionados (European Commission, 2010; Evans et al., 2013). No obstante, la aceptación de los alimentos genéticamente modificados (AGM) es un tema complejo, la falta de conocimientos elementales sobre los procesos biotecnológicos que llevan a su producción y sobre sus posibles riesgos y beneficios son la base sobre la cual se debe abordar esta problemática.

La Organización de las Naciones Unidas para la Alimentación y la Agricultura (FAO 2019, Green Facts 2019), indica que en los cultivos transgénicos (CT), cuya finalidad es la alimentación, no mostró daños al medio ambiente ni a la salud en ninguna parte del mundo (Winter y Gallegos, 2006). De hecho, la reducción en el uso de pesticidas y herbicidas que conlleva el uso de transgénicos se traduce en beneficios medioambientales y para la salud de los productores del 
Julio Gabriel Ortega, Cinthia Carchi Carch, Karla Morán Nieto...

campo (FAO, 2019). Hasta ahora, en los países donde se produjo CT, no hubo ningún informe verificable de que causen algún peligro importante para la salud o el medioambiente. Por el contrario, se están viendo algunos beneficios sociales y ambientales. Los agricultores están empleando menos plaguicidas y están sustituyendo productos químicos tóxicos con otros menos nocivos. Como consecuencia de ello, los productores agrícolas y los suministros de agua están protegidos de los venenos, y aves e insectos benéficos están volviendo a los campos de los productores agrícolas (FAO, 2019).

Existe un amplio consenso científico en que los OMGs que se encuentran actualmente en el mercado no representan un peligro mayor que los alimentos convencionales (König, et al., 2004, AAAS, 2012) y hasta la fecha no se documentó ningún caso de enfermedad en humanos debido al consumo de OGMs (Key et al., 2008). De hecho, mientras que los nuevos alimentos producidos por técnicas convencionales, la inocuidad de las modificaciones raramente es evaluada, todos los OGMs deben someterse a controles exhaustivos para garantizar su inocuidad, tanto para la salud humana como para el medioambiente antes de ser comercializados (Quan y Thomas, 2004; Key et al., 2008).

Ningún OGM presenta riesgo alguno para la salud (por la producción de moléculas no deseadas, o por provocar alergias) o el medio ambiente (por la diseminación no deseada de genes). Varias organizaciones científicas internacionales, y notablemente el Consejo Internacional para la Ciencia afirman que los OGMs comercializados no son peligrosos para la salud humana y que los riesgos de diseminación de OGMs en el medio ambiente son correctamente controlados. Otras organizaciones no científicas, entre ellas el polémico comité de investigación independiente sobre ingeniería genética de origen francés (Séralini et al., 2012), el Panel de Ciencia Independiente, del Reino Unido, alegan que los estudios realizados por los organismos acreditados son insuficientes o superficiales (Lendman, 2017) y que en los CT se deben tomar las prevenciones pertinentes para evitar la contaminación genética del medio ambiente (Heald y Smith, 2006; Shipitalo et al. 2008).

En Ecuador no se autorizó la liberación al ambiente, la experimentación, el uso o la comercialización de ningún organismo o producto transgénico. En enero del año 2000 varias organizaciones de la sociedad civil detectaron el ingreso de un barco que traía una carga de 30000 toneladas métricas (TM) de torta de soya importada de Estados Unidos enmarcada en el programa PL480. Se recolectaron muestras de soya y a través de exámenes de laboratorio se comprobó que ésta era transgénica. Ante estas evidencias se interpuso un recurso de amparo constitucional. El triunfo de este recurso de amparo sentó un precedente jurídico a través del cual es ilegal la propagación en el medio ambiente, experimentación, uso, comercialización e importación de alimentos transgénicos, hasta que no haya una regulación (Manzur et al., 2014).

Asimismo, en Ecuador de las normas jurídicas más importantes es el de la Constitución de la República, la cual en su artículo 401 menciona: "Se declara al Ecuador libre de cultivos y semillas transgénicas" (Bravo y León, 2013). Excepcionalmente, y sólo en caso de interés nacional debidamente fundamentado por la Presidencia de la República y aprobado por la Asamblea Nacional, se podrán introducir semillas y cultivos genéticamente modificados. El Estado regulará bajo estrictas normas de bioseguridad, el uso y el desarrollo de la biotecnología moderna y sus productos, así como su experimentación, uso y comercialización. Se prohíbe la aplicación de biotecnologías riesgosas o experimentales. Además, existen otros artículos donde exclusivamente se protege al país de cualquier riesgo que atente contra la biodiversidad nacional.

Asimismo, la Ley Orgánica del Régimen de la Soberanía Alimentaria (LORSA) publicada el año 2009 y modificada el año 2010, donde el párrafo 2 del artículo 26 menciona; "Las materias primas 
que contengan insumos de origen transgénico únicamente podrán ser importadas y procesadas, siempre y cuando cumplan con los requisitos de sanidad e inocuidad, y que su capacidad de reproducción sea inhabilitada, respetando el principio de precaución, de modo que no atenten contra la salud humana, la soberanía alimentaria y los ecosistemas. Los productos elaborados en base a transgénicos serán etiquetados de acuerdo a la ley que regula la defensa del consumidor".

Sin embargo, se dio por sentado la completa oposición a la introducción de los alimentos transgénicos como un deseo generalizado de la población, sin estudios que demuestren esa tendencia en las actitudes de los ecuatorianos frente a los alimentos transgénicos. Por lo expuesto, se ha creído importante, realizar la presente investigación que permita determinar si la sociedad civil y comunidad universitaria de la UNESUM están informados sobre los beneficios y riesgos de los ATs, así como también conocer el nivel de aceptación de los mismos.

La UNESUM a través de sus Facultades y Carreras como la de Agropecuaria, viene implementado una nueva malla curricular de estudios, en la que se incluyen los proyectos integradores de saberes (PIS), estos proyectos tienen el propósito de articular y fortalecer la docencia, la investigación con los estudiantes y la vinculación con la sociedad. Es propósito del PIS velar por el rigor científico en la generación de conocimientos y tecnología que sea aplicables y cuyos productos sean transferidos a la sociedad civil. En este entendido se vio por pertinente investigar con los estudiantes de las asignaturas de Biología Molecular, Genética y Bioestadística del cuarto y quinto nivel respectivamente de la malla curricular, la situación de la venta y consumo de alimentos provenientes de OTs en Jipijapa, Porto Viejo y Manta. El estudio pretendió analizar, entre otros aspectos, el nivel de conocimiento, las actitudes y las preferencias de los alimentos transgénicos por parte del consumidor.

Por lo mencionado, los objetivos de la presente investigación fueron: i) determinar la presencia de ATs en cadenas de supermercados y negocios, ii) conocer la precepción de la sociedad civil acerca de los ATs y iii) determinar la percepción de la comunidad universitaria acerca de los ATs.

\section{DESARROLLO}

\section{MATERIALES Y MÉTODOS}

Este estudio fue realizado en el periodo Marzo a Septiembre 2019. En el mismo participaron estudiantes de cuarto y quinto nivel de las asignaturas de Biología Molecular, Genética y Bioestadística respectivamente, de la Carrera de Ingeniería Agropecuaria de la Facultad de Ciencias Naturales y de la Agricultura, como parte de los Proyectos Integradores de Saberes (PIS) que se vienen implementando.

Se establecieron cómo lugares de estudio las grandes cadenas de supermercados como El Comisariato, TIA, AKI y otras tiendas y frigoríficos de barrio de Jipijapa, Manta y Portoviejo. Asimismo, se encuesto a personas de la sociedad civil y la comunidad universitaria de las 13 Carreras de la Universidad Estatal del Sur de Manabí (UNESUM).

\section{Metodología}

Preparación de la encuesta

La encuesta fue elaborada participativamente entre el profesor y los estudiantes. Esta encuesta estuvo compuesta de 13 preguntas. En la encuesta participaron 84 estudiantes de las asignaturas antes mencionadas.

\section{Visita a los Centro Comerciales}

Para la visita a los centros comerciales como el Comisariato, AKI, TIA y tiendas de barrio se pidió permiso a los gerentes, subgerentes o dueños de los comercios visitados. 
Julio Gabriel Ortega, Cinthia Carchi Carch, Karla Morán Nieto...

Los estudiantes de la asignatura de Biología Molecular "A" visitaron estos centros comerciales en grupos de 3 a 4 personas, para revisar todos los productos que contenían ATs, a los cuales se les tomó los datos como el nombre del producto, los ingredientes transgénicos que contiene, la fecha de caducidad y otros datos relevantes. Finalmente se sacaron fotografías de cada producto analizado.

\section{Encuesta a la sociedad civil}

Para determinar la percepción de la sociedad civil acerca de los ATs, fueron organizados 10 grupos (dos estudiantes/grupo) de la asignatura de Biología Molecular "B", donde cada grupo realizó 25 encuestas a la sociedad civil, totalizando unas 250 encuestas. Sin embargo, se realizó un tratamiento de la calidad de los datos, lo que permitió la selección de 130 encuestas válidas.

\section{Encuesta a la comunidad universitaria}

Para determinar la percepción de la comunidad universitaria acerca de los productos transgénicos, fueron organizados 17 grupos (dos estudiantes/grupo) de la asignatura de Genética y

Bioestadística, de la Carrera Agropecuaria, donde cada grupo realizó 25 encuestas a estudiantes de 15 carreras de cuatro facultades de la UNESUM (www.unesum.edu.ec) y dos grupos de nivelación, totalizando unas 425 encuestas.

\section{Análisis estadístico.}

Se analizaron las distintas variables del estudio mediante el cálculo de estadísticos descriptivos básicos. Las variables cualitativas, tanto las nominales o categóricas como las ordinales, fueron descritas con las frecuencias absolutas y porcentajes de cada una de las categorías o de los valores ordenados. Para la comparación de dos o más muestras independientes, estando medida tanto la variable dependiente o de respuesta como los factores de estudio de forma cualitativa, se elaboraron tablas de contingencia, que utilizando la prueba de estadística no paramétrica de ChiCuadrada, fueron contrastados con la hipótesis nula, por lo que se estableció que las variables que componen las tablas son independientes, estableciéndose la significancia al p<0.05 de probabilidad entre el valor observado y esperado de las tablas de contingencia. Estos análisis fueron realizados mediante el software estadístico SPSS (SPSS, 2006).

\section{RESULTADOS}

\section{Alimentos transgénicos encontrados}

Se observó más de una veintena de productos de una amplia variedad de alimentos que contienen algún compuesto transgénico. Estos van desde snacks, galletas, salsa, embutidos, etc. como se detalla en la Tabla 1.

Tabla 1. Alimentos transgénicos que se comercializan en el Comisariato, AKI, TIA y tiendas de barrio. Jipijapa, 2019.

\begin{tabular}{clll}
\hline Número & Producto & Empresa & \\
\hline 1 & Palomitas para microondas & & Contiene transgénicos \\
2 & Maní Cris & Contiene transgénicos \\
3 & Cheese tris & Frito Lay & Contiene transgénicos \\
4 & Cheetos & Frito Lay & Contiene transgénicos \\
5 & Mortadela Tipo II & Plumrose & Contiene transgénicos \\
6 & Filete de pollo asado & Plumrose & Contiene transgénicos \\
7 & Hamburguesa asada & Plumrose & Contiene transgénicos \\
\hline
\end{tabular}




\begin{tabular}{clll}
\hline 8 & Jamón Tipo II & Plumrose & Contiene transgénicos \\
9 & Salchichas Tipo II & Plumrose & Contiene transgénicos \\
10 & Salchichas de pollo & Plumrose & Contiene transgénicos \\
11 & Salchichas de ternero & Plumrose & Contiene transgénicos \\
12 & Picaditas & Plumrose & Contiene transgénicos \\
13 & Nuggets de pollo & Plumrose & Contiene transgénicos \\
14 & Salchipollo & Mr. Pollo & Contiene transgénicos \\
15 & Jamón de pollo & Mr. Pollo & Contiene transgénicos \\
16 & Mortadela de pollo & Mr. Pollo & Contiene transgénicos \\
17 & Chorizo de pollo & Mr. Pollo & Contiene transgénicos \\
18 & Tocino Tipo I & Piggis & Contiene transgénicos \\
19 & Salsa china de soya & Oriental & Contiene transgénicos \\
20 & Salsa Inglesa & Oriental & Contiene transgénicos \\
\hline
\end{tabular}

En la tabla 2, se observan algunos productos comercializados por MI COMISARIATO, tanto en Jipijapa y Manta.

Tabla 2: productos que contienen transgénicos comercializados por el Comisariato

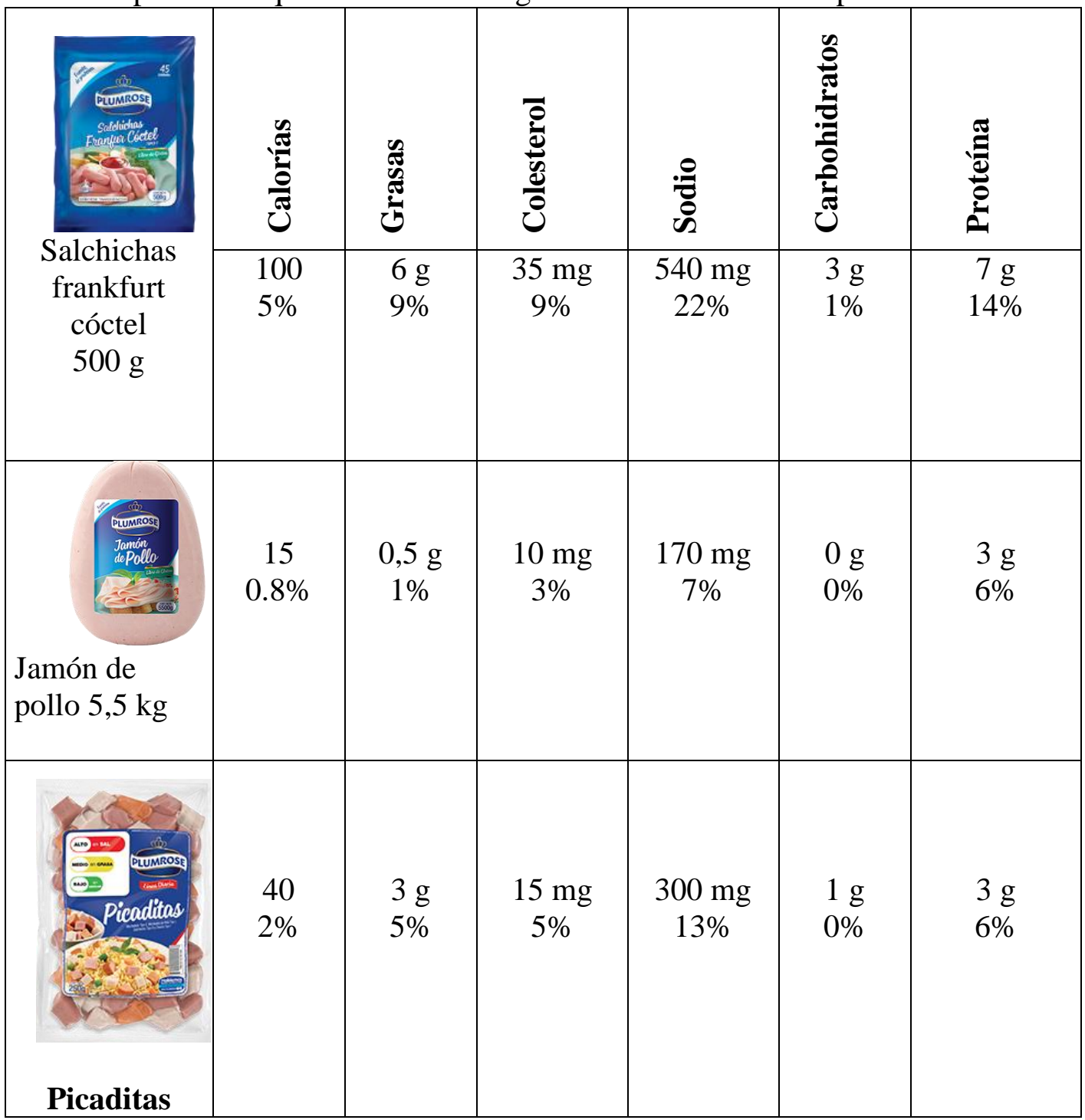




\begin{tabular}{|l|l|l|l|l|l|l|}
\hline $250 \mathrm{~g}$ & & & & & & \\
\hline
\end{tabular}

Los productos encontrados son alimentos básicos que la población consume. Se observó que en muchos de los casos las etiquetas eran poco visibles y les faltaba detallar el porcentaje de contenido del ingrediente transgénico en el alimento.

También se observó que las empresas y tiendas de barrio no hacen ninguna explicación sobre la existencia y venta de estos productos. Asimismo, no se observaron estrategias de marketing para motivar su consumo.

A continuación se presentan imágenes de algunos productos encontrados en el comercio de los supermercados y tiendas de barrio.

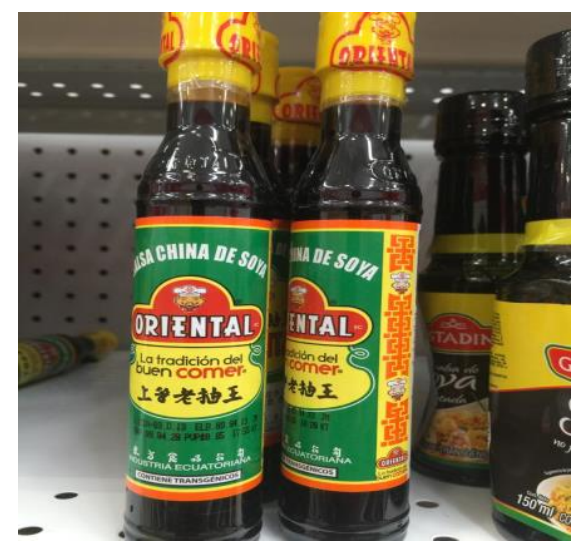

Figura 1. Salsa china

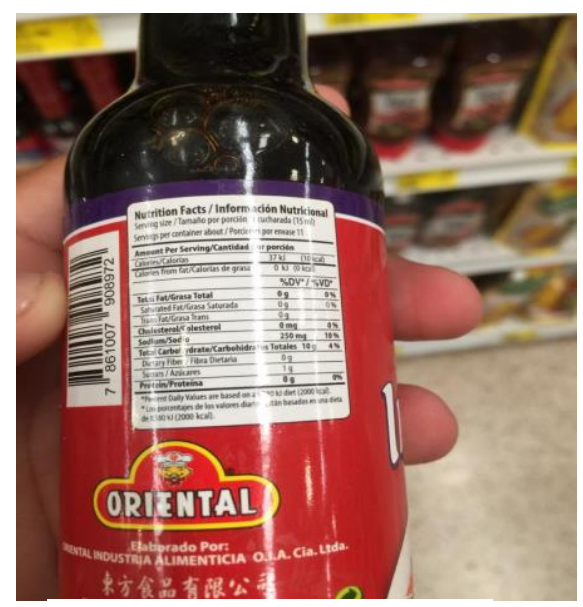

Figura 3. Salsa soya oriental

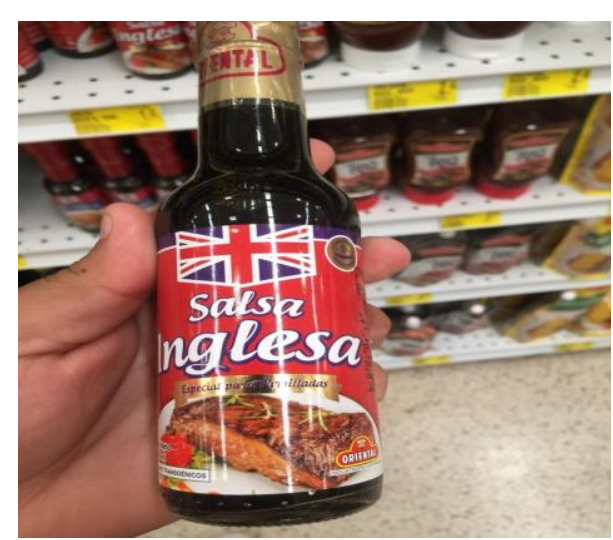

Figura 2. Salsa inglesa

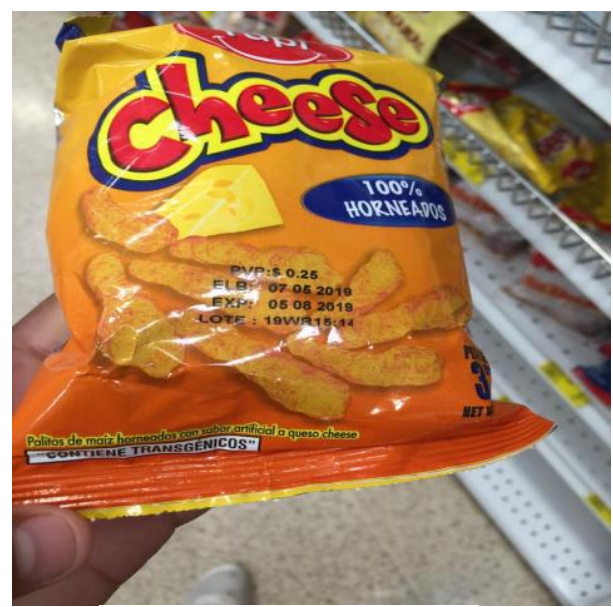

Figura 4. Cheese 




Figura 5. Galletas ritz

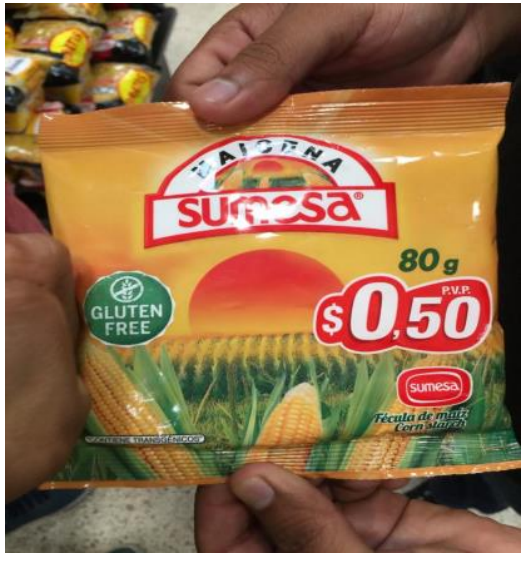

Figura 7. Maicena

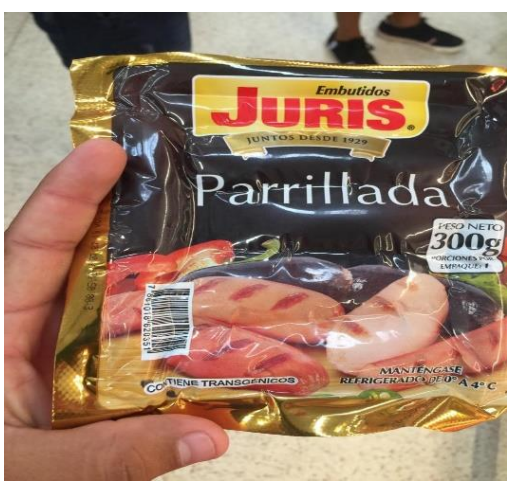

Figura 9. Embutidos

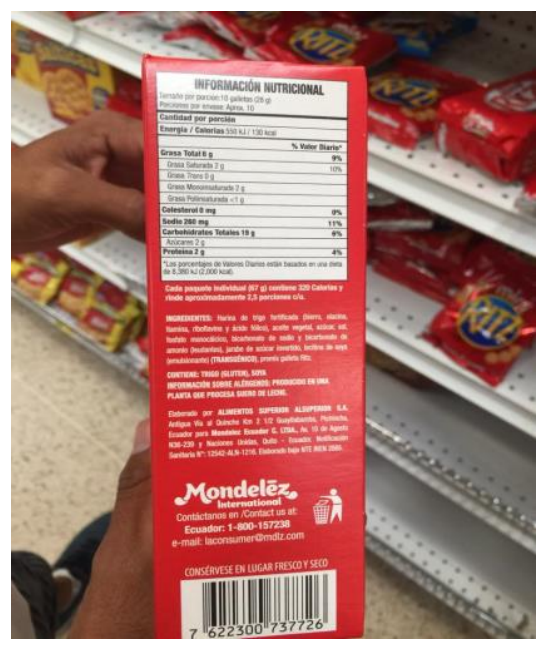

Figura 6. Chorizo

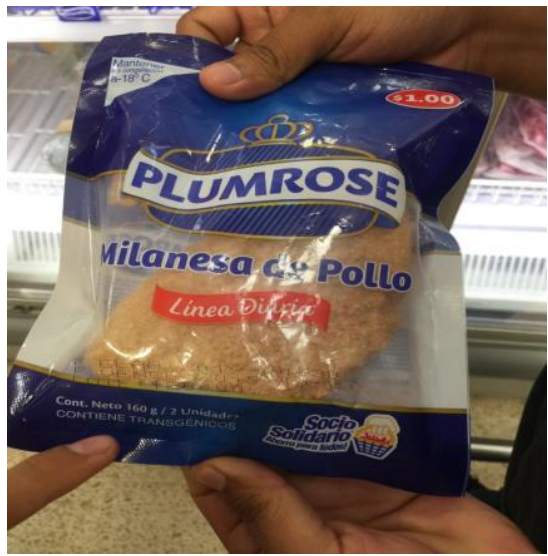

Figura 8. Milanesa de pollo

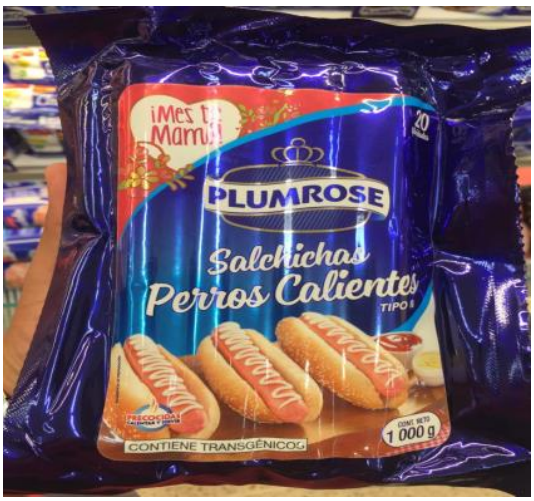

Figura 10. Salchichas 


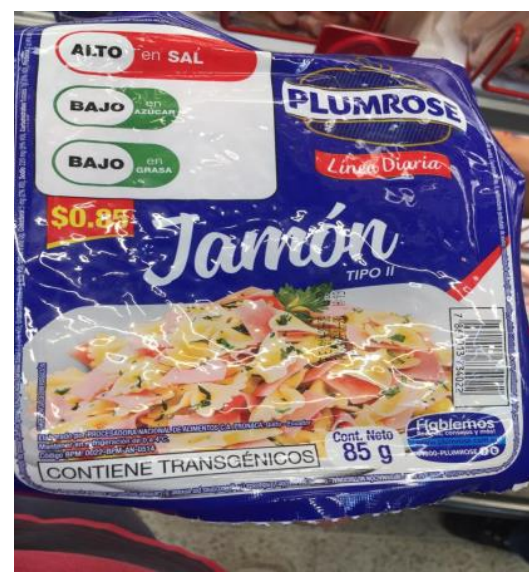

Figura 11. Jamòn

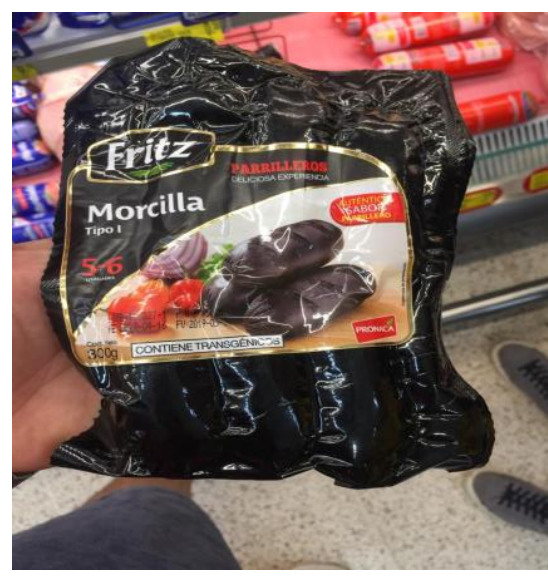

Figura 12. Morcilla



Figura 14. Bebida de soya



Figura 12. Mortadela

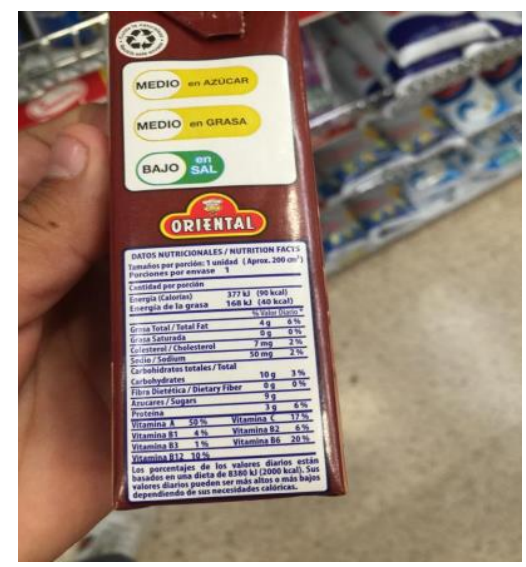

Figura 13. Galletas

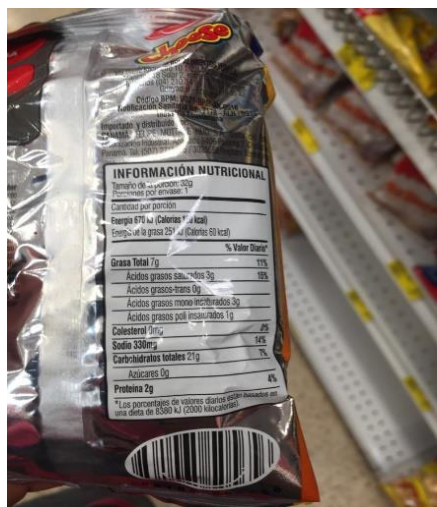

Figura 15. Cheese tris 


\section{Análisis de las encuestas a la sociedad civil}

Análisis de las distribuciones

En la Tabla 1, se muestran los principales parámetros estadísticos para cada una de las variables nominales obtenidas en las preguntas formuladas.

Tabla 1. Estadísticos para variables nominales de preguntas realizadas a la sociedad civil.

\begin{tabular}{|l|r|r|r|r|r|}
\hline & \multicolumn{7}{|c|}{ Preguntas } \\
\cline { 2 - 7 } Estadísticos & \multicolumn{1}{|c|}{2} & 3 & 6 & \multicolumn{1}{c|}{8} \\
\hline Desv. típ. &, 500 &, 500 &, 741 &, 502 & 1,057 \\
Varianza &, 250 &, 250 &, 549 &, 252 & 1,117 \\
Asimetría &, 156 &, 156 &, 062 &,- 062 &, 057 \\
Curtosis & $-2,007$ & $-2,007$ & $-1,161$ & $-2,028$ & $-1,281$ \\
\hline
\end{tabular}

2. ¿Cuál es su rango de edad?

3. ¿A qué área académica perteneces?

6. Indique las ventaja que cree que tienen los alimentos transgénicos sobre los alimentos convencionales

7. Indique las desventajas que cree que tienen los alimentos transgénicos sobre los alimentos convencionales

8. Indique porque cree que se permite la venta de transgénicos

La tabla 1 refleja que los datos obtenidos no tienen distribución normal para las preguntas 2, 3, 6, 7 y 8.

En la Tabla 2 se muestran los principales parámetros estadísticos para cada una de las variables ordinales obtenidas en las preguntas formuladas.

Tabla 2. Estadísticos para variables ordinales de preguntas realizadas a la sociedad civil.

\begin{tabular}{|l|r|r|r|r|r|r|r|r|}
\hline & \multicolumn{10}{|c|}{ Preguntas } \\
\cline { 2 - 9 } Estadísticos & \multicolumn{1}{|c|}{1} & \multicolumn{1}{c|}{4} & \multicolumn{1}{c|}{5} & \multicolumn{1}{c|}{9} & \multicolumn{1}{c|}{10} & \multicolumn{1}{c|}{11} & \multicolumn{1}{c|}{412} & 135 \\
\hline Desv. típ. &, 500 &, 500 &, 741 &, 502 & 1,057 & 1,076 & 1,293 &, 864 \\
Varianza &, 250 &, 250 &, 549 &, 252 & 1,117 & 1,158 & 1,672 &, 747 \\
Asimetría &, 156 &, 156 &, 062 &,- 062 &, 057 &,- 142 & 1,576 & 1,732 \\
Curtosis & $-2,007$ & $-2,007$ & $-1,161$ & $-2,028$ & $-1,281$ & $-1,251$ &, 836 & 1,782 \\
\hline
\end{tabular}

1. ¿A qué sexo pertenece?

4. ¿Sabe usted que son los alimentos transgénicos?

5. ¿Sabe usted como identificar los alimentos transgénicos?

9. ¿Qué considera mayor en los alimentos transgénicos, los beneficios o los perjuicios?

10. ¿Comprarías alimentos transgénicos?

11. Conque frecuencia come productos transgénicos

12. ¿El consumidor tiene derecho a decidir si come o no alimentos transgénicos?

13. ¿Las empresas deben informar en sus empaques - etiquetas que sus productos contienen transgénicos?

La tabla 2 refleja que los datos obtenidos no tienen distribución normal para las preguntas 1, 4, 5 , 91011,12 y 13.

Análisis de las encuestas

En la encuesta de la sociedad civil participaron 53\% del género masculino y $46 \%$ del femenino. De los cuales el 34\% fueron personas con edades entre los 18 a 21 años, $21 \%$ entre 22 a 25 años y $18 \%$ entre 26 a 30 años. En general el $73 \%$ de los entrevistados fueron jóvenes. Del total de personas entrevistadas el $68 \%$ tenían formación básica y profesional y 32\% fueron otros (obreros y amas de casa).

Según la encuesta realizada de 130 personas encuestadas el 54\% conoce que son los ATs De las 130 personas encuestadas, 35\% indicaron a que los ATs son más baratos, $21 \%$ se conservan mejor, 19\% tienen mejor aspecto de presentación (Tabla 3); y el $48 \%$ de los entrevistados consideraron a los ATs como beneficiosos para su consumo (Tabla 3).

Asimismo, la encuesta mostró que el 34\% de los entrevistados consideran a los ATs como perjudiciales a la salud y $30 \%$ lo consideran perjudiciales al ambiente (Tabla 3 ). 
El 37\% de los entrevistados considera que los ATs son permitidos para su venta por los intereses económicos, $12 \%$ por presión internacional y $12 \%$ por petición de los consumidores (Tabla 3). El 30\% de los encuestados opinó que sí compraría los ATs y 34\% mencionó entre si y otros no comprarían los ATs (Tabla 3). Pero quedo claro para el 74\% de los encuestados que tienen el derecho de decidir para consumir o no los ATs (Tabla 10), a esto va asociado a que el $73 \%$ opina que los ATs deben tener etiquetadas de identificación visibles (Tabla 3).

Tabla 3. Frecuencias, porcentajes y análisis de Chi-cuadrada al $\mathrm{P}<0,01$ de probabilidad para 13 preguntas en la sociedad civil.

\begin{tabular}{|c|c|c|c|}
\hline Preguntas & Frecuencia & Porcentaje válido & $\mathbf{P}>0.01$ \\
\hline \multicolumn{4}{|l|}{ Género } \\
\hline masculino & 70 & 53,8 & \\
\hline femenino & 60 & 46,2 & \\
\hline Total & 130 & 100,0 & 0,38 \\
\hline \multicolumn{4}{|l|}{ Edad } \\
\hline $15-17$ & 14 & 10,8 & \\
\hline $18-21$ & 44 & 33,8 & \\
\hline $22-25$ & 27 & 20,8 & \\
\hline $26-30$ & 24 & 18,5 & \\
\hline mayor a 31 & 21 & 16,2 & \\
\hline Total & 130 & 100,0 & 0,00 \\
\hline \multicolumn{4}{|l|}{ Formaciòn } \\
\hline Ciencias básicas y ambientales & 31 & 23,8 & \\
\hline Ciencias de la salud & 16 & 12,3 & \\
\hline Ciencias sociales y humanidades & 12 & 9,2 & \\
\hline Ingeniería & 13 & 10,0 & \\
\hline Economía y negocios & 16 & 12,3 & \\
\hline Otros & 42 & 32,3 & \\
\hline Total & 130 & 100,0 & 0,00 \\
\hline \multicolumn{4}{|l|}{ Conocimiento } \\
\hline $\mathrm{Si}$ & 70 & 53,8 & \\
\hline No & 60 & 46,2 & \\
\hline Total & 130 & 100,0 & 0,38 \\
\hline \multicolumn{4}{|l|}{ Identificación } \\
\hline $\mathrm{Si}$ & 38 & 29,2 & \\
\hline No & 59 & 45,4 & \\
\hline algunos & 33 & 25,4 & \\
\hline Total & 130 & 100,0 & 0,01 \\
\hline \multicolumn{4}{|l|}{ Ventajas } \\
\hline Beneficios nutricionales & 12 & 11,0 & \\
\hline Mejor aspecto & 21 & 19,3 & \\
\hline Mejor sabor & 13 & 11,9 & \\
\hline Mayor conservación & 23 & 21,1 & \\
\hline Màs baratos & 38 & 34,9 & \\
\hline Mas ecològicos & 2 & 1,8 & \\
\hline Total & 109 & 100,0 & 0,00 \\
\hline \multicolumn{4}{|l|}{ Desventajas } \\
\hline perjudicial para la salud & 42 & 34,4 & \\
\hline peor sabor & 11 & 9,0 & \\
\hline màs caros & 21 & 17,2 & \\
\hline perjudica al ambiente & 37 & 30,3 & \\
\hline perjudica a la economia & 11 & 9,0 & \\
\hline Total & 122 & 100,0 & 0,00 \\
\hline \multicolumn{4}{|l|}{ Causas de venta } \\
\hline presiòn del sector agropecuario & 15 & 11,5 & \\
\hline presiòn internacional & 16 & 12,3 & \\
\hline informes cientìficos & 12 & 9,2 & \\
\hline intereses econòmicos & 48 & 36,9 & \\
\hline son saludables & 8 & 6,2 & \\
\hline
\end{tabular}

66 UNESUM-Ciencias. Publicación cuatrimestral. Vol. 4, Año 2020, No. 1 (Enero - Abril) 


\begin{tabular}{lccc}
\hline peticiòn de los consumidores & 16 & 12,3 & \\
interès politico & 11 & 8,5 & \\
peticiòn de grupos ecologistas & 4 & 3,1 & 0,73 \\
Total & 130 & 100,0 & \\
\hline Beneficios o perjuicios & & & \\
\hline beneficio & 63 & 48,5 & \\
perjuicio & 67 & 51,5 & \\
Total & 130 & 100,0 & \\
\hline Compra & & & \\
\hline Si & 40 & 30,8 & \\
No & 27 & 20,8 & \\
algunos si, otros no & 45 & 34,6 & \\
no se & 18 & 13,8 & \\
Total & 130 & 100,0 & \\
\hline Derecho a decidir & & & \\
\hline Si & 96 & 73,8 & \\
No & 9 & 6,9 & \\
algunos si, otros no & 1 &, 8 & \\
no se & 17 & 13,1 & \\
Total & 7 & 5,4 & \\
\hline Etiquetado & & 73,8 & \\
\hline si & 96 & 10,8 & \\
no & 14 & 10,8 & \\
algunos si, otros no & 14 & 4,6 & \\
no se & 6 & & \\
Total & 130 & & \\
\hline
\end{tabular}

\section{Análisis de significancia}

El análisis de Chi-cuadra al $\mathrm{P}<0.01$ de probabilidad (Tabla 3), mostró diferencias altamente significativas entre lo observado y esperado para las preguntas $2,3,5,6,7,8,10,12$ y 13 , denotando que al menos un criterio fue diferente estadísticamente.

\section{Análisis de las encuestas a la comunidad universitaria}

\section{Análisis de las distribuciones}

En la Tabla 4, se muestran los principales parámetros estadísticos para cada una de las variables nominales obtenidas en las preguntas formuladas.

Tabla 4. Estadísticos para variables nominales de preguntas realizadas a la academia.

\begin{tabular}{|l|r|r|r|r|r|}
\hline \multirow{2}{*}{ Estadísticos } & \multicolumn{5}{|c|}{ Preguntas } \\
\cline { 2 - 6 } & \multicolumn{1}{|c|}{2} & \multicolumn{1}{|c|}{. 3} & \multicolumn{1}{c|}{. } & \multicolumn{1}{c|}{8} \\
\hline Desv. típ. &, 756 & 1,545 & 1,344 & 1,368 & 1,817 \\
Varianza &, 572 & 2,386 & 1,805 & 1,872 & 3,302 \\
Asimetría &, 372 &, 088 &,- 526 &, 609 &, 168 \\
Curtosis &, 156 & $-1,148$ &,- 369 & $-1,059$ &,- 388 \\
\hline
\end{tabular}

Ver el significado de los números de las preguntas en la tabla 1.

La tabla 13 refleja que los datos obtenidos no tienen distribución normal para las preguntas 2, 3, 6,7 y 8 .

En la Tabla 5, se muestran los principales parámetros estadísticos para cada una de las variables ordinales obtenidas en las preguntas formuladas.

Tabla 5. Estadísticos para variables ordinales de preguntas realizadas a la academia.

\begin{tabular}{|c|c|c|c|c|c|c|c|c|}
\hline \multirow[b]{2}{*}{ Estadísticos } & \multicolumn{8}{|c|}{ Preguntas } \\
\hline & 1 & 4 & 5 & 9 & 10 & 11 & 412 & 135 \\
\hline Desv. típ. & , 493 & ,471 & ,759 & ,436 & 1,017 & 1,105 & 947 & 1,103 \\
\hline Varianza & ,243 & ,221 & ,577 & 190 & 1,034 & 1,221 & ,896 & 1,216 \\
\hline Asimetría & 360 &,- 728 & 272 & 1,142 &,- 026 &,- 319 & 1,512 & 1,079 \\
\hline
\end{tabular}


Julio Gabriel Ortega, Cinthia Carchi Carch, Karla Morán Nieto...

\begin{tabular}{|l|r|r|r|r|r|r|r|r|}
\hline Curtosis & $-1,879$ & $-1,476$ & $-1,222$ &,- 702 & $-1,291$ & $-1,343$ & 1,029 &,- 425 \\
\hline
\end{tabular}

La tabla 5, refleja que los datos obtenidos no tienen distribución normal para las preguntas 1, 4, $5,91011,12$ y 13.

Análisis de las encuestas

En la encuesta de la comunidad universitaria de la UNESUM participaron 59\% del género masculino y $41 \%$ del femenino. De los cuales el $44 \%$ fueron personas con edades entre los 18 a 21 años, $42 \%$ entre 22 a 25 años y $9 \%$ entre 26 a 30 años. En general el $95 \%$ de los entrevistados fueron jóvenes. Las 425 personas entrevistadas pertenecían a las cuatro facultades de la UNESUM y a nivelación (Tabla 6).

Según la encuesta realizada, de 425 personas encuestadas el $67 \%$ no conoce que son los ATs (Tabla 15), y el $40 \%$ no sabe cómo identificarlos (Tabla 6).

De las 425 personas encuestadas, $45 \%$ indicaron que los ATs tienen mayor conservación y 16\% son más baratos (Tabla 6); y el $75 \%$ de los entrevistados consideran los ATs como beneficiosos para su consumo (Tabla 6).

Asimismo, la encuesta mostró que el $51 \%$ de los entrevistados consideran a los ATs como perjudiciales a la salud, $21 \%$ lo consideran más caros y $14 \%$ perjudiciales al ambiente (Tabla 6). El $42 \%$ de los entrevistados considera que los transgénicos son permitidos para su venta por los intereses económicos, $14 \%$ por petición de los consumidores y $12 \%$ por presión internacional (Tabla 6).

El 40\% de los encuestados opinó que algunos si, otros no compraría los ATs y $31 \%$ mencionó entre si compraría y $18 \%$ que no comprarían los ATs (Tabla 6). Pero quedo claro para el $67 \%$ de los encuestados indicaron que tienen el derecho de decidir para consumir o no los ATs (Tabla 22), a esto va asociado a que el $63 \%$ opina que los ATs deben tener etiquetadas de identificación visibles (Tabla 6).

Análisis de significancia

El análisis de Chi-cuadrada al $\mathrm{P}<0.01$ de probabilidad (Tabla 7), mostró diferencias altamente significativas entre lo observado y esperado para todas las preguntas, denotando que al menos un criterio fue diferente estadísticamente.

Tabla 7. Análisis de frecuencias, porcentajes y Chi-cuadrada al $\mathrm{P}<0,01$ de probabilidad para 13 preguntas.

\begin{tabular}{lccc}
\hline \multicolumn{1}{c}{ Preguntas } & Frecuencia & Porcentaje válido & P>0.01 \\
\hline Género & & & \\
masculino & 250 & 48,8 & 0,00 \\
femenino & 175 & 1,2 & \\
Total & 425 & & \\
\hline Edad & & 4,2 & \\
\hline $15-17$ & 18 & 43,5 & \\
$18-21$ & 185 & 41,9 & \\
$22-25$ & 178 & 9,4 & \\
$26-30$ & 40 &, 9 & \\
mayor a 31 & 4 & 100,0 & \\
Total & 425 & 18,8 & \\
\hline Formaciòn & & 18,8 & \\
\hline Ciencias básicas y ambientales & 80 & 18,8 \\
Ciencias de la salud & 80 & 18,8 \\
Ciencias sociales y humanidades & 80 & 18,8 & \\
Ingeniería & 80 & 5,9 \\
Economía y negocios & 80 & 100,0 & 0,00 \\
Otros & 25 & & \\
Total & 425 & & \\
\hline
\end{tabular}




\begin{tabular}{|c|c|c|c|}
\hline \multicolumn{4}{|l|}{ Conocimiento } \\
\hline $\mathrm{Si}$ & 140 & 32,9 & \\
\hline No & 285 & 67,1 & \\
\hline Total & 425 & 100,0 & 0,00 \\
\hline \multicolumn{4}{|l|}{ Identificación } \\
\hline $\mathrm{Si}$ & 161 & 37,9 & \\
\hline No & 170 & 40,0 & \\
\hline algunos & 94 & 22,1 & \\
\hline Total & 425 & 100,0 & 0,00 \\
\hline \multicolumn{4}{|l|}{ Ventajas } \\
\hline Beneficios nutricionales & 45 & 11,3 & \\
\hline Mejor aspecto & 44 & 11,0 & \\
\hline Mejor sabor & 41 & 10,3 & \\
\hline Mayor conservación & 182 & 45,6 & \\
\hline Màs baratos & 64 & 16,0 & \\
\hline Mas ecològicos & 23 & 5,8 & \\
\hline Total & 399 & 100,0 & 0,00 \\
\hline \multicolumn{4}{|l|}{ Desventajas } \\
\hline perjudicial para la salud & 201 & 51,1 & \\
\hline peor sabor & 23 & 5,9 & \\
\hline màs caros & 86 & 21,9 & \\
\hline perjudica al ambiente & 57 & 14,5 & \\
\hline perjudica a la economia & 26 & 6,6 & \\
\hline Total & 393 & 100,0 & 0,00 \\
\hline \multicolumn{4}{|l|}{ Causas de venta } \\
\hline presiòn del sector agropecuario & 42 & 10,1 & \\
\hline presiòn internacional & 49 & 11,8 & \\
\hline informes cientìficos & 24 & 5,8 & \\
\hline intereses econòmicos & 176 & 42,5 & \\
\hline son saludables & 22 & 5,3 & \\
\hline peticiòn de los consumidores & 59 & 14,3 & \\
\hline interès politico & 23 & 5,6 & \\
\hline peticiòn de grupos ecologistas & 19 & 4,6 & \\
\hline Total & 414 & 100,0 & 0,00 \\
\hline \multicolumn{4}{|l|}{ Beneficios o perjuicios } \\
\hline beneficio & 171 & 74,7 & \\
\hline perjuicio & 58 & 25,3 & \\
\hline Total & 229 & 100,0 & 0,00 \\
\hline \multicolumn{4}{|l|}{ Compra } \\
\hline $\mathrm{Si}$ & 134 & 31,5 & \\
\hline No & 78 & 18,4 & \\
\hline algunos si, otros no & 171 & 40,2 & \\
\hline no se & 42 & 9,9 & \\
\hline Total & 425 & 100,0 & 0.00 \\
\hline \multicolumn{4}{|l|}{ Derecho a decidir } \\
\hline $\mathrm{Si}$ & 285 & 67,1 & \\
\hline No & 70 & 16,5 & \\
\hline algunos si, otros no & 36 & 8,5 & \\
\hline no se & 34 & 8,0 & \\
\hline Total & 425 & 100,0 & 0,00 \\
\hline \multicolumn{4}{|l|}{ Etiquetado } \\
\hline si & 270 & 63,5 & \\
\hline no & 45 & 10,6 & \\
\hline algunos si, otros no & 56 & 13,2 & \\
\hline no se & 54 & 12,7 & \\
\hline Total & 425 & 100,0 & 0,00 \\
\hline
\end{tabular}

\section{DISCUSIÓN}

Un estudio realizado por Oleas et al. (2016) determinó que pocos adolescentes de la Provincia de Imbabura dijeron tener conocimientos sobre los ATs (18,3\%), y a pesar de que algunos escucharon 
Julio Gabriel Ortega, Cinthia Carchi Carch, Karla Morán Nieto...

el tema, no tienen una idea clara sobre este tipo de alimentos ya que apenas el 5,2\% los definen correctamente. Esto podría deberse a que es un tema muy poco difundido, debido a la controversia que ha ocasionado en los últimos años. En otros trabajos, como el realizado en Chile sobre 400 consumidores (Schnettler et al., 2009), encontraron que solo el 26,3\% de la muestra total indicó conocer lo que significa un alimento transgénico. En nuestro estudio se observó que más del 50\% de los jóvenes entrevistados de la sociedad civil y de la academia conocen a los ATs, y más del $50 \%$ saben identificarlos.

Por otra parte, Viedma et al. (2014), en un estudio realizado en España con el fin de conocer cuál es el nivel de conocimiento del ciudadano sobre algunos temas biológicos y genéticos, que muchas veces subyacen en las controversias sociales actuales y que pueden ser indicativo del porqué del rechazo o aceptación de determinados productos, se dio al consumidor la siguiente afirmación para que indique si la considera verdadera o falsa: los tomates comunes no tienen genes, mientras que los transgénicos sí. El 47,4\% de la población estudiada desconoce que la afirmación propuesta es falsa, lo que nos indica que casi la mitad de la población tiene carencias básicas en temas biológicos y genéticos, esto dificulta que puedan tener criterios claros a la hora de la aceptación o rechazo de los Alimentos Genéticamente Modificado (AGM).

Por los resultados encontrados en nuestro estudio, podríamos inferir que el nivel de compresión y conocimiento sobre los alimentos transgénicos en el Ecuador ha cambiado en los últimos años.

Por otra parte, en el estudio realizado por Viedma et al. (2014), con el propósito de indagar más sobre el nivel de conocimiento del consumidor en el tema de los transgénicos y su repercusión sobre la salud del consumidor, se da al consumidor la siguiente afirmación: si una persona come transgénicos, los genes de esta persona resultan modificados, con la intención de que diga si es verdadera o falsa. El $40 \%$ de los consumidores desconocen si los genes se pueden ver o no modificados y el 3,6\% consideran cierta la afirmación.

Entre los motivos por los cuales los adolescentes consideraron que se producen alimentos transgénicos en el estudio de Rodríguez y Sayadi (2008) y Oleas et al. (2016) y señalaron: los intereses económicos (10\%), para mejorar los problemas nutricionales $(6,1 \%)$ y para combatir el hambre (4,3\%). En nuestro estudio, pudimos determinar que ambos grupos encuestados (sociedad civil y académico) consideran que los ATs son importantes para su venta por los intereses económicos, por presión internacional y por la petición de los consumidores.

Por una parte, en el estudio de Oleas et al. (2016), los ATs fueron considerados beneficiosos porque se conservan más tiempo $(8,4 \%)$ y por otra piensan que son malos porque aportan pocos nutrientes $(8,5 \%)$. En nuestro estudio la percepción de ambos grupos encuestados indicaron un criterio similar al mencionado y los ATs son beneficiosos para el consumo, pero al preguntar sobre los beneficios o perjuicios de los ATs indicaron que estos son perjudiciales para la salud y el medioambiente. Además, la sociedad civil le da más importancia a que los ATs porque son más baratos, y la academia opinó que lo más importante sería la mayor conservación de los ATs.

La polémica generada en torno al tema de los AGM está motivada por el miedo a lo nuevo que siempre resulta desconocido. Distintos autores hablaron de los posibles beneficios de estos alimentos y otros centraron su discurso en los riesgos potenciales (Viedma et al. 2014).

Es importante considerar que a pesar de la prohibición de los alimentos transgénicos, en Ecuador existen más de 70 productos transgénicos disponibles en los mercados sin que los consumidores conozcan que se trata de este tipo de alimentos. En el estudio de Oleas et al. (2016), el 11,9\% de 
los adolescentes manifestaron que no conocían la existencia de AT en nuestro país, mientras que del 6,4\% que si lo conocían, el 4,9\% señalaron que provenían de otros países. En nuestro estudio el conocimiento de los ATs fue mayor a los reportados.

Estudios realizados por el Centro de Investigaciones Sociológicas (CIS) en 2001 sobre biotecnología en la población española planteaban al consumidor la pregunta de si sería capaz de consumir un transgénico y sólo el $28,8 \%$ de la población dijo que sí los consumiría. Si lo comparamos con un estudio similar realizado también por el CIS en 1996, el porcentaje de quienes sí consumirían transgénicos fue mayor (40\%), ése retroceso en la aceptación de los transgénicos en la población española podía estar relacionado con la información recibida por el consumidor (Luján, 2004). También mencionaron que si comprarían los ATs y que tienen el derecho de decidir para consumirlos. Pero ambos grupos enfatizaron que las empresas están en la obligación de que sus productos y/o ATs deben estar etiquetados visiblemente.

Si bien es cierto que los hallazgos de nuestro estudio acerca de la aceptación de los alimentos transgénicos, se suma a la tendencia de la mayoría de la población mundial. Aun se observa cierta resistencia y esta radica en el desconocimiento de todo lo concerniente a estos alimentos y por ende, a la falta de compresión de los posibles riesgos y beneficios de los mismos. A esto se suma la ausencia de estrategias públicas dirigidas a dar información científica y veraz a la población para que puedan o no tomar la decisión de aceptar los ATs, libre de mitos que sobredimensionen los riesgos y fuera de engaños que prometan beneficios inalcanzables.

Está claro que es importante tener estrategias públicas que establezcan un sistema nacional que regule de manera efectiva las prácticas de ingeniería genética, que controle el ingreso y la producción de OGM, y que también permita a la ciudadanía tener información real acerca de los alimentos que consume a diario.

La población estudiada mostró un conocimiento medio y aceptación a los ATs y una alta predisposición para obtener mayor información, por lo que es importante que el sector educativo incluya en los contenidos de formación este tema, para que los consumidores integren y refuercen sus conocimientos y puedan hacer un razonamiento crítico.

\section{CONCLUSIONES}

En general, se aprecia un cierto nivel de conocimiento de los alimentos transgénicos por parte de los consumidores, aunque aún existe un grupo considerable que los desconoce totalmente.

Por otra parte, aunque en el mercado existen alimentos que indican en su etiquetado que contienen ingredientes modificados genéticamente, la mayoría de los consumidores creen, o bien que sí consumieron alimentos transgénicos en alguna ocasión, o bien que desconocen con certeza no haberlos consumido, quedando así de manifiesto la sospecha de la transparencia del mercado respecto a las producciones transgénicas. En nuestro estudio las actitudes y preferencias sociales hacia los alimentos modificados genéticamente indican que los consumidores, a pesar de las ventajas ofrecidas por los alimentos transgénicos, continúan prefiriendo, en general, los convencionales. A partir del análisis de la importancia relativa, se desprende que los dos atributos más importantes en las preferencias de los consumidores fueron, en este orden, el medioambiente y la salud. Destaca, igualmente, que el precio parece ser el factor de mayor importancia para los consumidores a la hora de adquirir alimentos transgénicos.

De los resultados hallados, algunas recomendaciones podrían hacerse de las que destacarían las siguientes:

- Las empresas deberían ofrecer mayor información a la sociedad mediante el diseño de un plan estratégico sobre las producciones transgénicas, de modo que la población conozca las 
Julio Gabriel Ortega, Cinthia Carchi Carch, Karla Morán Nieto...

particularidades que diferencian a este tipo de alimentos y sepan cómo identificarlos en el mercado.

- Estrategias de marketing basadas en alegaciones beneficiosas para la salud humana (enriquecimiento en vitaminas, ácido fólico, Omega-3, etc.) podrían favorecer el potencial futuro del mercado de los ATs de segunda generación.

\section{REFERÉNCIAS BIBLIOGRÁFICAS}

American Association for the Advancement of Science (AAAS), Board of Directors (2012). Legally Mandating GM Food Labels Could Mislead and Falsely Alarm Consumers.

Argenbio (2019). $\quad \mathrm{La} \quad$ ingeniería $\quad$ genética. Disponible en http://www.argenbio.org/index.php?action=novedades\&note $=151$

Bravo, E.; León, X. (2013). Monitoreo participativo del maíz ecuatoriano para detectar la presencia de proteínas transgénicas. La Granja. Vol. 17(1): 16-24.

CHILEBIO (2019). Mitos y realidades: la aplicación de la biotecnología para producir alimentos genéticamente modificados (gm) es diferente a los sistemas convencionales de producción de cultivos. Disponible en http://www.chilebio.cl/mitos_realidades.php

European Commission. (2010) Biotechnology Report; Special Eurobarometer. Wave 73.1. TNS Opinion \& Social. Disponible en https://ec.europa.eu/commfrontoffice/publicopinion/archives/ebs/ebs 341_en.pdf

Evans, E.; Ballen, F. (2013). A Synopsis of US Consumer perception of Genetically Modified (Biotech) Crops. Extension Data Information Spurce (EDIS) Insitute of Food and Agricultural Sciences. University of Florida.

FAO (2019). Repercusiones de los cultivos transgénicos en la salud y el medio ambiente. Depósito de documentos de la fao». Disponible en. Http://www.fao.org/3/y5160s/y5160s10.htm

Gabriel, J. (2014), Biotecnología moderna en los alimentos actuales y del mañana. J Selva Andina Biosph. 2014; 2(1):23-29. Disponible en http://www.scielo.org.bo/pdf/jsab/v2n1/v2n1_a04.pdf

Gabriel, J., Indacochea, B.; Valverde, Y; Narváez, W. (2018). Modern biotechnology in food. Adv Plants Agric Res. 8(6):476-477. DOI: 10.15406/apar.2018.08.00369

Gaskell, G.; Stares, S.; Allansdottir, A.; Allum, N.; Corchero, C.; Fischler, C.; Hampel, J.; Jackson, J.; Kronberger, N.; Mejlgaard, N.; Revuelta, G.; Schreiner, C.; Torgersen, H.; Wagner, W. (2006). Brussels: Europeans and Biotechnology in 2005: Patterns and Trends; 2006 Jul. 88. Final Report on Euro barometer 64.3. Disponible en https://ec.europa.eu/commfrontoffice/publicopinion/archives/ebs/ebs_244b_en.pdf

Green Facts (2019) Consenso Científico sobre los Cultivos Transgénicos y OMG. "El Estado Mundial de la Agricultura y la Alimentación 2003-2004". Disponible en https://www.greenfacts.org/es/omg/index.htm.

Heald, P.J.; Smith, J.C. (2006), The problem of social cost in a genetically modified. Hastings j: 87-151.

ISAAA (2019). Brief 54: global status of commercialized biotech/gm crops: 2018. Disponible en http://www.isaaa.org/resources/publications/briefs/54/default.asp

Jurkiewicz, A.; Zagórski, J.; Bujak, F.; Lachowski, S.; Florek Luszczki, M. (2014). Emotional attitudes of young people completing secondary schools towards genetic modification of organisms (GMO) and genetically modified foods (GMF). Ann Agric Environ Med. 21(1): 205-211.

Key, S.; K-C Ma, J.; Drake, P (2008). Genetically modified plants and human health. J R Soc Med 2008: 101: 290298. DOI 10.1258/jrsm.2008.070372

König, A.; A.Cockburn, A.; R.W.R.Crevel, R.; ; Grafstroem, R.; Hammerling, Kimber, U.; Knudsen, I.; H.A.Kuiper, H.; Peijnenburg, A.; Penninks, H.; Poulsen, M.; Schauzu, H. Wal, J. (2004). Assessment of the safety of foods derived from genetically modified (GM) crops. Food and Chemical Toxicology 42 (7): 1047-1088. https://doi.org/10.1016/j.fct.2004.02.019.

Lendman, S. (2017) Potential Health Hazards of Genetically Engineered Foods. Global Research. Disponible en https://www.globalresearch.ca/potential-health-hazards-of-genetically-engineered-foods/8148.

Luján, J.L. (2004). Sobre las imágenes sociales de la ciencia. Ciencia en general frente a aplicaciones concretas en el caso de la biotecnología. Sistema 279(180): 123-132. Disponible en https://scielo.conicyt.cl/scielo.php?script=sci nlinks\&ref=129158\&pid=S1726$\underline{569 X 201500020001300014 \& \operatorname{lng}=\mathrm{es}}$.

72 UNESUM-Ciencias. Publicación cuatrimestral. Vol. 4, Año 2020, No. 1 (Enero - Abril) 
Manzur, M.; Bravo, E.; Altieri, M.; Catacora, G.; Cárcamo, M. (2014). América Latina: la transgénesis de un continente. Visión crítica de una expansión descontrolada. 2da ed. Uruguay: Ediciones Böll. 190 p.

Oleas, M.E.; Tejada, E.E.; Lascano, R.M. (2016). Conocimientos y aceptación de alimentos transgénicos en adolescentes de la provincia de Imbabura, Ecuador. Rev Esp Nutr Comunitaria 22(1): 1 - 9.

Petit glossaire (2019). OGM, INRA. Disponible en http://www.toulouse.inra.fr/Presse/Communiques-de-presse/MaisOGM/(key)/5

Quan, L. and Thomas, W. (2004). Effects of information on consumers' willingness to pay for GM-corn-fed beef. Journal of Agricultural and Food Industrial Organization 2 (2): 1-16.

Rodriguez, M.; Sayadi, S. (2008). ¿Conocen los consumidores los alimentos transgénicos?: El efecto de sus cualidades en la opción de consumo? Páginas 90-94 in III Congreso Calidad Alimentaria, Instituto Andaluz de Investigación y Formación Agraria (I.F.A.P.A), Área de Economía y Sociología Agraria, Castilla y Leòn, España.

Schnettler B, Sepúlveda O, Ruiz F. (2009). Conocimiento y aceptación de alimentos genéticamente modificados en consumidores de la IX Región de Chile. Idesia 27(2):5-15. http://dx.doi.org/10.4067/S071834292009000200001 .

Scott, F. (2013). Evolution and Domestication: Selection on Developmental Genes? Developmental Biology (10 edición). Sinauer Associates Inc. ISBN 978-0-87893-978-7.

Séralini, G.E., Clair, E., Mesnage, R.; Gress, S.; Defarge, N.; Malatesta, M.; Hennequin, D.; Spiroux de Vendômois, J. (2012). Long term toxicity of a Roundup herbicide and a Roundup-tolerant genetically modified maize. Food and Chemical Toxicology http://dx.doi.org/10.1016/j.fct.2012.08.005

Shipitalo, M.J.; Malone, R.W.; Owens, L.B. (2008). IMPACT OF GLYPHOSATE-TOLERANT SOYBEAN AND GLUFOSINATE-TOLERANT CORN PRODUCTION ON HERBICIDE LOSSES IN SURFACE RUNOFF. J Environ Qual. 37(2):401- 408. HTTP://DX.DOI: 10.2134/JEQ2006.0540.

Traynor P, Adonis M, Gil L. (2007). Strategic approaches to informing the public about biotechnology in Latin America. Electron. J. Biotechnol. 10(2):169 - 177.

Viedma, I.; López, J.M; Serrano, M. y Balanza, S. (2014). Actitud del consumidor frente a los Alimentos Genéticamente Modificados. Technoscience 4(2): 1-15.

Winter, C.K. and Gallegos, L.K. (2006). Safety of Genetically Engineered Food. University of California Agriculture and Natural Resources Communications, Publication 8180.

Zilberman, D.; Kaplan, S.; Kim, E.; Hochman, G.; Graf, G. (2013). Continents divided Understanding differences between Europe and North America in acceptance of GM crops. GM crops \& food. 2013; 4(3), 202-208. 